1 Detection of conspicuous and cryptic food by marmosets (Callithrix jacchus): An evaluation of the importance of color and shape cues.

4 Priscilla Kelly Silva Barros ${ }^{\mathbf{a}},{ }^{\mathbf{b}}$; Felipe Nalon Castro ${ }^{\mathbf{c}}$; Daniel Marques Almeida

$5 \quad$ Pessoa*a,, b

6 a Laboratory of Sensory Ecology, Department of Physiology and Behavior, Biosciences

7 Center, Federal University of Rio Grande do Norte, Natal - RN, Brazil, 59072-970

8 brimate Center, Department of Physiology and Behavior, Biosciences Center, Federal

9 University of Rio Grande do Norte, Natal - RN, Brazil, 59072-970

10 'Laboratory of Human Behavior Evolution, Department of Physiology and Behavior,

11 Biosciences Center, Federal University of Rio Grande do Norte, Natal - RN, Brazil, $12 \quad 59072-970$

13 *Corresponding author: Av. Senador Salgado Filho, s/n. UFRN - Campus Universitario 14 Lago Nova. Bairro Lagoa Nova. UFRN, CB, DFS. Natal - RN, Brazil. 59078-970. Email: 15 daniel.pessoa@ufrn.br

17 Declarations of interest: The authors declare no competing or financial interests.

\title{
18 Highlights
}

- Shape cues alone improve capture of cryptic targets by female and male marmosets.

- The sole use of color cues leads to clear-cut foraging performances between sex.

21 - Females outperform males when searching for orange food against a green 22 background.

- Males outperform females when searching for green food against a green background.

- Males match females' performance when searching for orange camouflaged food. 


\section{Abstract}

26 Since 19th century, the adaptive function of color vision for object identification has

27 fascinated science. Countless articles uncovered the advantages of trichromacy (i.e., color

28 vision expressed by most humans) in detecting yellowish/reddish targets against a

29 background of mature green leaves. Unfortunately, the same attention was not offered to

30 achromatic visual information, that had their physiological foundations much more

31 explored than their possible adaptive function. So far, mostly because of studies

32 conducted in humans, we know that achromatic cues might also play an important role in

33 object identification, particularly when camouflage is involved. For instance, dichromacy

34 (i.e., color vision expressed by many colorblind humans), favors the detection of

35 camouflaged targets by exploitation of shape cues. The present study sought to evaluate

36 the relative importance of color and shape cues on the detection of food targets by female

37 and male marmosets (Callithrix jacchus). Animals were observed with respect to their

38 foraging behavior and the number of food targets captured. We confirmed that females

39 are advantageous in detecting conspicuous food against a green background and revealed

40 that females and males rely on shape cues to segregate cryptic food. Unexpectedly, males

41 outperformed females in cryptic food foraging. Camouflage improved males' (but not

42 females') performance.

43 Key words: camouflage; chromatic noise; color vision; foraging; visual polymorphism;

44 Platyrrhine. 


\section{INTRODUCTION}

For an animal to have color vision, it must possess, at least, two distinct classes of photoreceptor cells in its retina, in addition to a neural network capable of comparing the responses of these cells (Cowey and Heywood 1995). Literature has shown that the greater the variety of retinal photoreceptor cells, the better the animal's ability to distinguish color (Bowmaker 1998). In this context, diurnal mammals exhibit dichromatic color vision (Jacobs 2018), associated with two photopigment classes, as observed in some colorblind humans. Yet, primates (Jacobs 2010) appear to have evolved three different types of cones in their retina. This results in trichromatic vision, like that found in humans with normal color vision.

The distribution of color vision among primates has a peculiar pattern. Uniform trichromacy is observed among Old World monkeys and apes (Catarrhini), in which most individuals within a population exhibit three different classes of cone photopigments: $\mathrm{S}$ pigments, which preferentially absorb short wavelengths (blue range); M pigments, which preferentially absorb medium wavelengths (green range); and L pigments, which preferentially absorb long wavelengths (red range). While the gene encoding the $\mathrm{S}$ pigment is found on an autosomal chromosome, $\mathrm{M}$ and $\mathrm{L}$ pigments are coded on the $\mathrm{X}$ chromosome (Jacobs 2010).

Alternatively, New World primates (Platyrrhini) display a visual polymorphism, characterized by the occurrence of females with trichromatic (heterozygosis) or dichromatic (homozygosis) vision, and obligatory dichromatic males (hemizygosis) (Hunt et al. 1993). This visual polymorphism occurs because a single gene encodes $\mathrm{M} / \mathrm{L}$ pigments, and is located on the $\mathrm{X}$ chromosome, in addition to a gene that encodes the $\mathrm{S}$ pigment on an autosomal chromosome (Carvalho et al. 2017). Different visual alleles can 
73 be found on the $\mathrm{X}$ chromosome, which produce photopigments with distinct light

74 absorption peaks, yielding trichromats with S, M and L cone pigments, and dichromats with S and only one M or L pigment.

On one hand, color is considered an important visual cue, since it facilitates the identification of objects and patterns within a scenario, as well as assisting in memorization (Wichmann et al. 2002). The visual recognition of objects can be improved by simply adding color to an image (Shevell and Kingdom 2008). Since the $19^{\text {th }}$ century, when the naturalist Grant Allen proposed, in 1879, that the evolution of fruit coloration and animal color vision had occurred in parallel, color vision in primates has been understood as resulting from an adaptive process involving the identification of conspicuously colored food resources (see Jacobs 2007). In line with that, the frugivory and folivory hypotheses were postulated to explain which advantages trichromats would have in detecting ripe fruit and young leaves, respectively, against a background of mature green leaves (Mollon 1989; Sumner and Mollon 2000; Dominy and Lucas 2001).

On the other hand, by using color to camouflage objects, that is, adding chromatic noise, one can hamper object identification, especially when other cues (e.g., size, shape, texture) are not available (Liebe et al. 2009). This happens because neural processing of chromatic and achromatic information occurs concurrently and competitively. Thus, as argued by Regan and collaborators (2001), primates with greater dependence on color cues (e.g., trichromats) should be more affected by chromatic noise, that is, they would

93 focus a significant amount of their neural processing to deal with chromatic information,

94 at the cost of a worse processing of achromatic cues. Alternatively, those primates with poorer color vision (e.g., dichromats) would benefit from a reduced chromatic noise, being able to redirect additional neural processing to achromatic information assessment, 
97 important for the identification of shapes, outlines, and textures. In fact, Mollon (1989)

98 predicted that, given their possible disadvantage in detecting conspicuous food items,

99 dichromats should compensate their inferior performance by identifying camouflaged

100 objects. Investigations presenting camouflaged geometric figures to humans (Morgan et

101 al. 1992; Saito et al. 2006) and non-human primates (Saito et al. 2005) showed that

102 dichromats, when compared with trichromats, were more capable of breaking camouflage

103 and detected hidden shapes. Indeed, some naturalistic/ecological studies gave support to

104 the idea that the visual polymorphism of Neotropical primates balances (Hiwatashi et al.

105 2010) the advantages of dichromats in recognizing hidden objects in the environment

106 (Caine et al. 2003; Melin et al. 2007; Smith et al. 2012) and those of trichromats in

107 detecting conspicuous targets (Caine and Mundy 2000; Smith et al. 2003; Melin et al.

108 2013, 2017; Pessoa et al. 2014; Abreu et al. 2019).

109 Research on Branisella boliviana, a 26 million-year-old Neotropical primate fossil,

110 weighing approximately 760 grams (Kay et al. 2002) and, possibly, exhibiting visual

111 polymorphism (Heesy and Ross 2001), suggests that leaves had never been a significant

112 component of the diet of Neotropical primate ancestors, which likely fed on fruits and

113 insects (Kay 1984). This leads to the inference that folivory has never been important in

114 the evolution of callitrichids (i.e., marmosets and tamarins), which are the smallest extant

115 Neotropical primates (weighting less than 800g; Arruda et al. 2019). In addition, it has

116 been proposed that insectivory and predation pressure are selective factors that

117 maintained the visual polymorphism in this taxon (Pessoa et al. 2014; De Moraes et al. 118 2021).

119 In the past decades, color vision of common marmosets (Callithirx jacchus) has

120 been substantially examined through theoretical, behavioral, electrophysiological and 
121 molecular approaches (Travis et al. 1988; Tovée et al. 1992; Hunt et al. 1993; Wilder et

122 al. 1996; Shyue et al. 1998; Kawamura et al. 2001; Surridge and Mundy 2002; Pessoa et

123 al. 2005a; Freitag and Pessoa 2012; Pessoa et al. 2014; Moreira et al. 2015; Abreu et al.

124 2019; Pessoa and Freitag 2019; Mantovani et al. 2020), showing that C. jacchus expresses

125 a tri-allelic visual polymorphism, that encodes $\mathrm{M} / \mathrm{L}$ pigments with maximum absorption

126 at $543 \mathrm{~nm}, 556 \mathrm{~nm}$ and $562 \mathrm{~nm}$, and allows the existence of six visual phenotypes (three

127 dichromats and three trichromats) in natural populations (Jacobs 2007).

128 Although many naturalistic studies have already addressed the possible advantages

129 and disadvantages of color vision in food identification (Caine and Mundy 2000; Caine

130 et al. 2003; Smith et al. 2003; Leonhardt et al. 2009; Freitag and Pessoa, 2012; Smith et

131 al. 2012; Bompas et al. 2013; Melin et al. 2013; Pessoa and Freitag, 2019), we believe

132 the relative importance of different visual information (e.g., shape and color) in food

133 target detection has not been sufficiently analyzed in non-human primates. This is due

134 because the attention of previous studies has fallen almost exclusively on controlling

135 stimuli's color, while shape has been overlooked. As such, the present study sought to

136 examine the influence of color and shape cues on food detection by female and male

137 marmosets (Callithrix jacchus). Here, for practical reasons, and since, at least, two thirds

138 of females are expected to be trichromats (Jacobs 2007), while the totality of males are

139 dichromats, we assume that females, as a group, will behave as trichromats, while males

140 will perform as dichromats, an approach that has been successfully adopted by behavioral

141 ecologists when visual phenotypes are unknown (Dominy et al. 2003, Yamashita et al.

142 2005). We hypothesize that chromatic (i.e., color) and achromatic (i.e., shape) signals, as

143 well as chromatic and achromatic noise, will influence food detection by female and male

144 marmosets. We also theorize that animals of different sex will display contrasting 
145 performances under conditions in which color cues play a decisive role in food

146 advertisement. Our predictions are that, when foraging on a background of dappled green

147 cubical elements (i.e., in the presence of lower chromatic noise and higher achromatic

148 noise): (1) females will outperform males when searching for orange cubical food items

149 (i.e., when color cues are available only for trichromatic animals), but not for green

150 (cryptic condition) or blue (i.e., when color cues are available for trichromats and

151 dichromats) cubical targets; (2) females and males will segregate green spherical targets

152 (i.e., when shape cues are available for all subjects) much easier than green cubical ones

153 (cryptic condition); and (3) females will outperform males when searching for orange

154 spherical items (i.e., when color and shape cues are available for trichromats, while only

155 shape cues are available for dichromats). Otherwise, when foraging on a background of

156 dappled green and orange cubical elements (i.e., in the presence of higher chromatic noise

157 and lower achromatic noise): (4) males will outperform females when searching for

158 orange spherical items (i.e., when shape cues will be available for dichromats, while will

159 be masked for trichromats by chromatic noise).

160

\section{MATERIAL AND METHODS}

\subsection{Subjects}

From a breeding colony of, approximately, 200 common marmosets (Callithrix

164 jacchus), maintained by the Federal University of Rio Grande do Norte's Primate Center,

165 we selected five family groups to take part in the experiments. Females and males of

166 different age classes were monitored, but only data from adults, subadults and juveniles

167 were analyzed in the present study, totaling 10 females and 15 males. All behavioral 
168

experiments were conducted in 2010 so, since we did not collect tissue samples from our subjects by then, determination of individual color vision phenotypes was not possible. To avoid the stress of daily capture and transportation to a new environment, family group had access to experimental enclosures ( $1.0 \mathrm{~m}$ width $\times 2.0 \mathrm{~m}$ length $\times 2.0 \mathrm{~m}$ height), containing platforms and branches of different heights, that was adjacent to their larger home enclosures ( $2.0 \mathrm{~m}$ width $\times 2.0 \mathrm{~m}$ length $\times 2.0 \mathrm{~m}$ height). Temperature and sunlight varied freely, according to local weather. Experimental sessions were always conducted in the mornings (07:00 a.m. to 10:00 a.m.), when food trays were removed from the enclosures. Water was always provided ad libitum. The experimental procedures were approved by the Animal Research Ethics Committee of the Federal University of Rio Grande do Norte (Proc.045/2009) and adhered to the legal requirements of Brazilian regulation. Experiments complied with the ARRIVE guidelines and were carried out in accordance with the National Institutes of Health guide for the care and use of Laboratory animals (NIH Publications No. 8023, revised 1978).

\subsection{Stimuli}

We produced visual targets using cake frosting (Arcolor), with the following composition: sugar, vegetable fat, vegetable gums, and glucose syrup. Food targets were molded into spheres or cubes (approximately 1 centimeter wide) and colored with food dyes (Arcolor), consisting of glucose syrup, modified starch, humectant propylene glycol, water, preservatives, and organic dyes. For each target hue (i.e., blue, green, or orange) we produced two variants with different brightness levels (e.g., light, and dark blue targets). We also produced background elements from white foam rubber sheets (EVA - Ethylene Vinyl Acetate) that were cut into small cubes (about $1 \mathrm{~cm}^{3}$ ), divided into five similar piles, and coated with different water-based paints, creating four 
192 different shades of green and one shade of orange. Once finished, the surface of food

193 targets and background elements appeared matte and polished. Using food targets and

194 background elements of the same size, same texture and variable brightness levels was a

195 strategy adopted by us to preclude the identification of food targets based on visual cues

196 other than color and/or shape.

197 Fine adjustments in stimuli colors were performed by a human trichromat

198 (P.K.S.B.) and a human dichromat (D.M.A.P.), to ensure that, on a green dappled

199 background, green-colored targets were cryptic and blue-colored targets were

200 conspicuous. These human subjects also ensured that orange-colored targets had the

201 same appearance as orange-colored background elements. Orange-colored targets were

202 also adjusted to seem conspicuous to trichromats (P.K.S.B.) and cryptic to dichromats

203 (D.M.A.P.), when viewed against a green dappled background.

\subsection{Spectral Measurements}

We measured stimuli's coloration with a portable spectrometer (USB4000 UV-

VIS Fiber Optic Spectrometer, Ocean Optics Inc.) running Spectra Suite software

207 (Ocean Optics Inc.), connected to a bifurcated optical fiber (R400-7-VIS-NIR, Ocean

208 Optics Inc.), that was attached to a probe-holder (RPH-1, Ocean Optics Inc.). The

209 system was illuminated with an artificial light source (LS-1, Ocean Optics, Inc.). For

210 system calibration we measured light reflecting from a white standard surface (WS-1,

211 Ocean Optics Inc.), and obstructed light coming from the light source for determining

212 the black standard. After calibration, we measured the reflectance spectra (coloration) of

213 food targets and background elements, positioning the tip of the optical fiber $0.5 \mathrm{~cm}$

214 from the measured surface, always at an angle of $45^{\circ}$. The number of spectra averaged

215 to scan, and boxcar were always set to 10 and 5, respectively. For each kind of stimuli, 
216 we randomly picked four samples, measured their coloration, and generated an average

217 reflectance spectra curve (Figure 1).

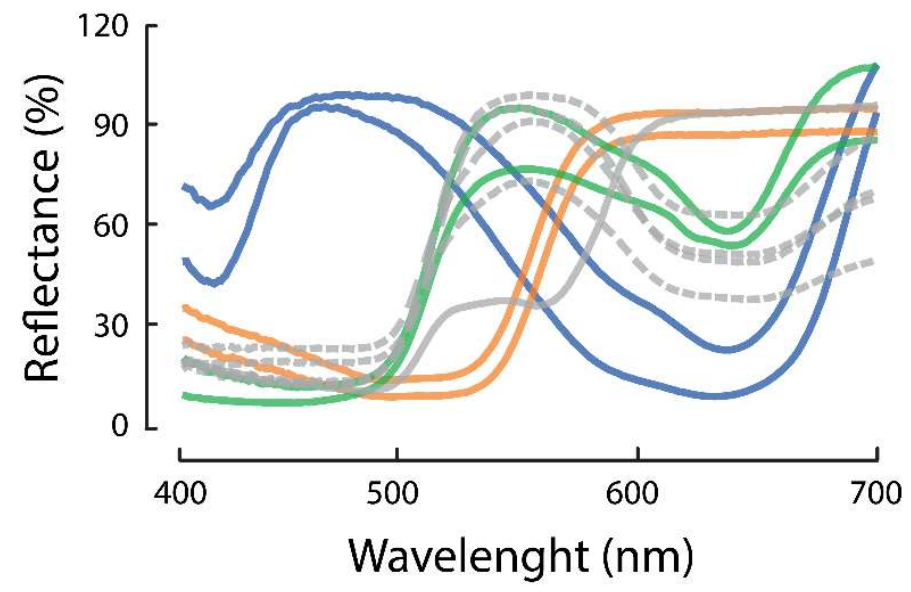

219 Figure 1. Stimuli reflectance spectra. Gray dashed lines represent the coloration of green background 220 elements (four different shades are shown), while the gray solid line illustrates the reflectance of orange background elements. Solid colored lines represent food targets coloration, in two different shades of blue

222 (curves peaking around $450 \mathrm{~nm}$ ), orange (curves peaking around $600 \mathrm{~nm}$ ) and green (curves peaking around $550 \mathrm{~nm})$.

\subsection{Visual Modeling}

Visual modeling was conducted in pavo 2.0 (Maia et al. 2019), a R package for

227 spectral analysis of color (R Core Team 2020). We started estimating the absolute amount

228 of light captured by marmosets' photoreceptors ('Qi' - quantum catch), considering three

229 factors: a) the reflectance spectrum of each stimulus (Figure 1); b) the illuminant spectrum

230 of enclosures' ambient light; c) and marmoset's visual sensitivity curves (for further

231 details see Perini et al. 2009). Then, we set the remaining parameters to default (i.e., trans

$232=$ 'ideal', vonkries $=$ FALSE, scale $=1$ ), and set relative $=$ FALSE, since we required

233 absolute quantum catches, instead of relative quantum catches. 
By generating MacLeod-Boynton chromaticity/luminance diagrams, which graphically represent how stimuli should excite marmosets' red-green, blue-yellow and

236 luminance visual channels, we modeled how trichromatic and dichromatic marmosets

237 would discriminate food targets from background elements. We constructed the diagrams

238 based on absolute quantum catch information for $\mathrm{S}\left(\mathrm{Q}_{\mathrm{S}}\right), \mathrm{M}\left(\mathrm{Q}_{\mathrm{M}}\right)$ and $\mathrm{L}\left(\mathrm{Q}_{\mathrm{L}}\right)$

239 photopigments. Since marmosets are polymorphic, and express three kinds of

240 trichromatic phenotypes (with S/M/L spectral peaks at 430/543/556 nm, 430/543/562 nm,

241 and 430/556/562 nm) and three kinds of dichromatic phenotypes (with S/L spectral peaks

242 at $430 / 543 \mathrm{~nm}, 430 / 556 \mathrm{~nm}$, and $430 / 562 \mathrm{~nm}$ ), we modeled chromaticity/luminance

243 diagrams for six different visual phenotypes. While trichromats exhibit three visual

244 channels, $\mathrm{Q}_{L} /\left(\mathrm{Q}_{\mathrm{M}}+\mathrm{Q}_{\mathrm{L}}\right)$ (i.e., red-green channel), $\mathrm{Q}_{\mathrm{S}} /\left(\mathrm{Q}_{\mathrm{M}}+\mathrm{Q}_{\mathrm{L}}\right)$ (i.e., blue-yellow channel),

245 and $\mathrm{Q}_{\mathrm{M}}+\mathrm{Q}_{\mathrm{L}}$ (i.e., luminance channel), dichromats only display two, a blue-yellow ( $\left.\mathrm{Q}_{\mathrm{S}} \mathrm{Q}_{\mathrm{L}}\right)$

246 and a luminance channel ( $\left.\mathrm{Q}_{\mathrm{L}}\right)$.

\subsection{Experimental procedure and behavioral observations}

We adjusted the number of food targets offered according to family size

(disregarding infants), preserving a ratio of two targets per animal (e.g., 10 targets were offered to a family composed of 5 animals). Then, we randomly scattered cubical or spherical targets among three, out of six, plastic trays (each measuring $30 \mathrm{~cm}$ width $\mathrm{x} 50$ cm length x $9 \mathrm{~cm}$ height), previously filled with green (lower chromatic noise conditions) or green and orange (higher chromatic noise condition) background elements, which we

254 placed on the floor of the experimental enclosures (Figure 2).

To each marmoset family group, we repeatedly presented (22 repetitions) six experimental setups (Figure 2): (A) Four shades of green background elements with two 
bioRxiv preprint doi: https://doi.org/10.1101/2021.04.11.439391; this version posted April 12, 2021. The copyright holder for this preprint (which was not certified by peer review) is the author/funder. All rights reserved. No reuse allowed without permission.

elements with two shades of orange cubical-shaped food targets; (C) Four shades of green background elements with two shades of green cubical-shaped food targets; (D) Four

260 shades of green background elements with two shades of orange spherical-shaped food

261 targets; (E) Four shades of green background elements with two shades of green

262 spherical-shaped food targets; (F) Two shades of green and one shade of orange

263 background elements with two shades of orange spherical-shaped food targets.
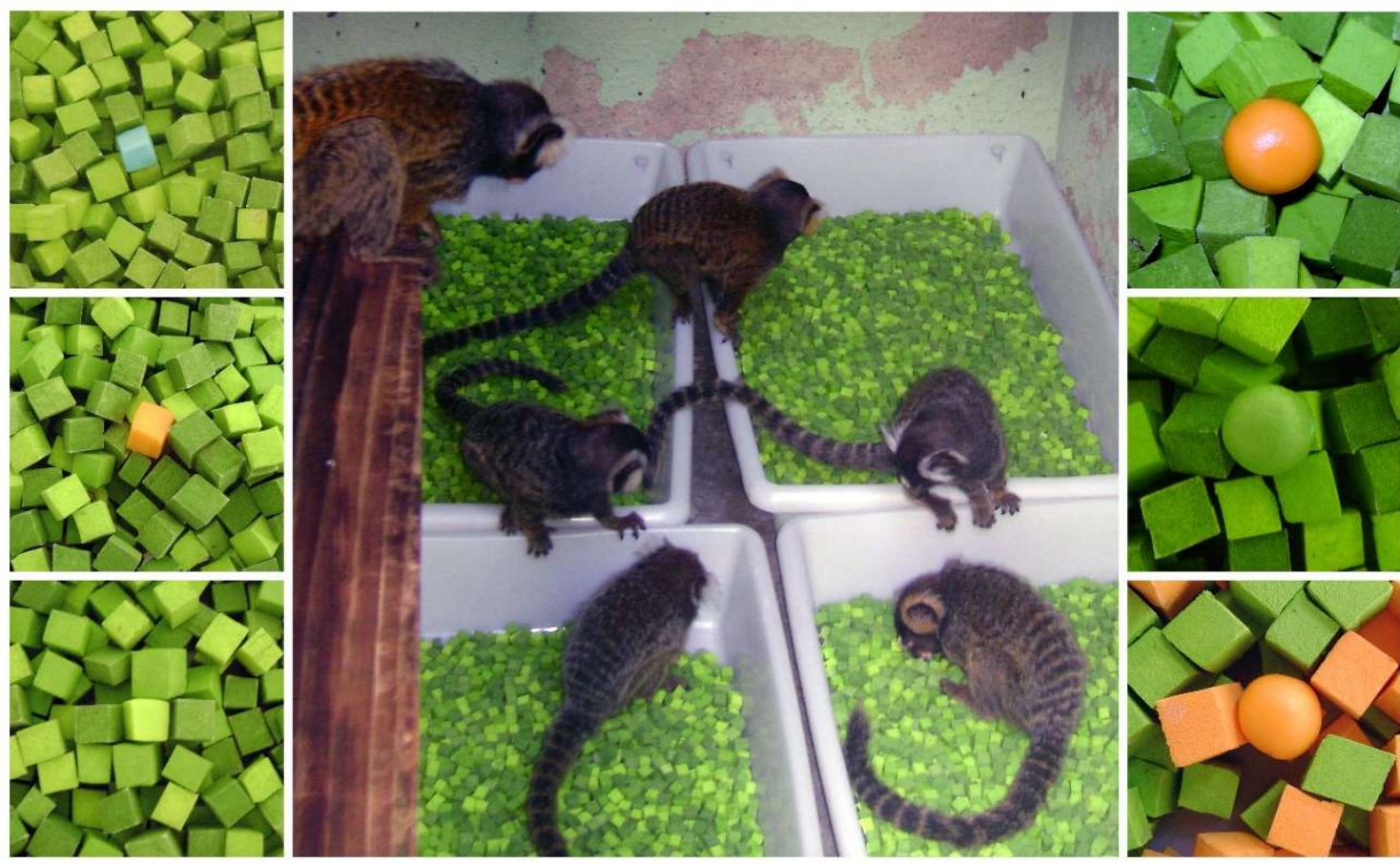

Figure 2. Subjects engaged in foraging activity (center photograph). Lateral panels illustrate six different setups used in the experiments. Upper left: green background elements with a blue cubical-shaped food target. Middle left: green background elements with an orange cubical-shaped food target. Bottom left: green background elements with a green cubical-shaped food target. Upper right: green background elements with an orange spherical-shaped food target. Middle right: green background elements with a green spherical-shaped food target. Bottom right: green/orange background elements with an orange spherical-shaped food target. 
experimentation enclosure. Presentation order for the six setups was randomized, as was

276 the time of the day for observing each family. On each experimental day, we exposed

277 each family to the same experimental setup, while pseudo-randomly selecting a focal

278 individual from each family (Altmann 1974) to be monitored regarding its foraging

279 behavior. Foraging behavior was defined as the visual scanning behavior directed towards

280 the plastic trays, as well as the behavior of actively searching (i.e., while on the plastic

281 trays, slowly walking and looking towards the stimuli) and capturing food targets. We did

282 not compute food manipulation and/or intake as foraging. Alongside, a second human

283 observer was responsible for using all-occurrence sampling (Altmann 1974) to record the

284 number of targets captured by every individual in the group.

285 We left infants out of the analysis since their behaviors were not registered. We were unable to register the foraging behavior (focal sessions) of one of the juvenile males,

287 although we recorded the number of targets captured by it in every experimental session. In addition, after we had already finished monitoring the foraging behavior (focal sessions) of one of the adult females, it fell ill and deceased, so the number of targets

290 captured by it could not be considered in our analysis. Except for these two incidents, every animal that managed to capture a food item was registered with respect to food acquisition. So, at the end of the study period, four complete focal sessions (measuring

293 foraging duration) had been monitored for each adult and subadult marmoset (ten females 294 and fourteen males), in a total of 16 hours of sampling effort (40 minutes for each focal 295 subject). In addition, for each family group, 22 sessions determining the number of targets captured were accomplished and included data from nine females and 15 males, in a total

297 of 220 minutes of sampling effort. We used two chronometers and two audio recorders 298 to register foraging information. 


\subsection{Statistical Analysis}

300

The performance of test subjects was analyzed according to their average foraging

301 time and average percentage of food targets captured. For calculating average foraging

302 time, we considered the total amount of time (in seconds) spent in foraging (while food

303 targets were still available) by each animal, dividing it by total session duration, which

304 ended when all food targets were consumed or after 10 minutes had passed. The average

305 percentage of food targets captured was determined considering the ratio between total

306 number of targets captured per animal and total number of targets offered for that specific

307 family group.

308 We ran six independent General Linear Models (GLM), with post-hoc Bonferroni,

309 for testing the effect of target shape, target color and background chromatic noise for each

310 of the dependent variables (i.e., average foraging time and average percentage of food

311 targets captured). We evaluated the effect of target shape by comparing experimental

312 setups (C) and (E), assigning target shape (round or square) and sex (female or male) as

313 independent variables. To explore the effect of target color, we compared experimental

314 setups (A), (B) and (C), selecting target color (orange, green or blue) and sex (female or

315 male) as independent variables. Finally, we explored the effect of background chromatic

316 noise by comparing experimental setups (D) and (F), defining background color (green

317 or orange-green) and sex (female or male) as independent variables. In all analyzes, the

318 alpha considered was 5\%.

3. RESULTS 
In chromaticity/luminance diagrams, stimuli similarity is given as a measure of spatial proximity, i.e., the further apart two stimuli are represented, the more dissimilar

324 they are, and vice-versa. With that said, Figures 3 and 4 predict, on one hand, that green 325 targets should be cryptic to dichromatic and trichromatic marmosets, since they overlap 326 with the green background elements with respect to chromaticity and luminance. But, on

327 the other hand, blue targets must be highly conspicuous against green background 328 elements to dichromatic and trichromatic individuals, when considering chromaticity, but not luminance. Figures 3 and 4 also predict that orange food targets should be cryptic to

330 dichromats and conspicuous to trichromats, since their chromaticity overlap with green

331 background elements on the blue-yellow channel, but not on the red-green visual channel.

332 In addition, compared to orange background elements, orange targets should always be cryptic.

\subsection{Influence of target color}

Target color influenced foraging time $\left(\mathrm{F}_{2,42}=16.90 ; \mathrm{p}<.001 ; \eta^{2}\right.$ Partial $\left.=0.446\right)$ and

336 percentage of targets captured $\left(F_{2,44}=3.88 ; p=.028 ; \eta^{2}\right.$ Partial $\left.=0.150\right)$. On one hand, both

337 female and male marmosets significantly invested more time foraging for green food,

338 when compared to their time spent looking for orange or blue items (Figure 5B). On the

339 other hand, females captured fewer green food items than orange ones, while males had

340 a bad time capturing orange food, which were significantly less captured than green and

341 blue targets, and a good time capturing blue targets, which were taken more frequently

342 than green and orange targets (Figure 5A).

343 We also found an interaction between target color and $\operatorname{sex}\left(\mathrm{F}_{2,44}=12.25 ; \mathrm{p}<.001\right.$;

$344 \eta^{2}$ Partial $\left.=0.358\right)$ for percentage of targets captured, with females capturing significantly

345 more orange food than males (Figure 5A). 

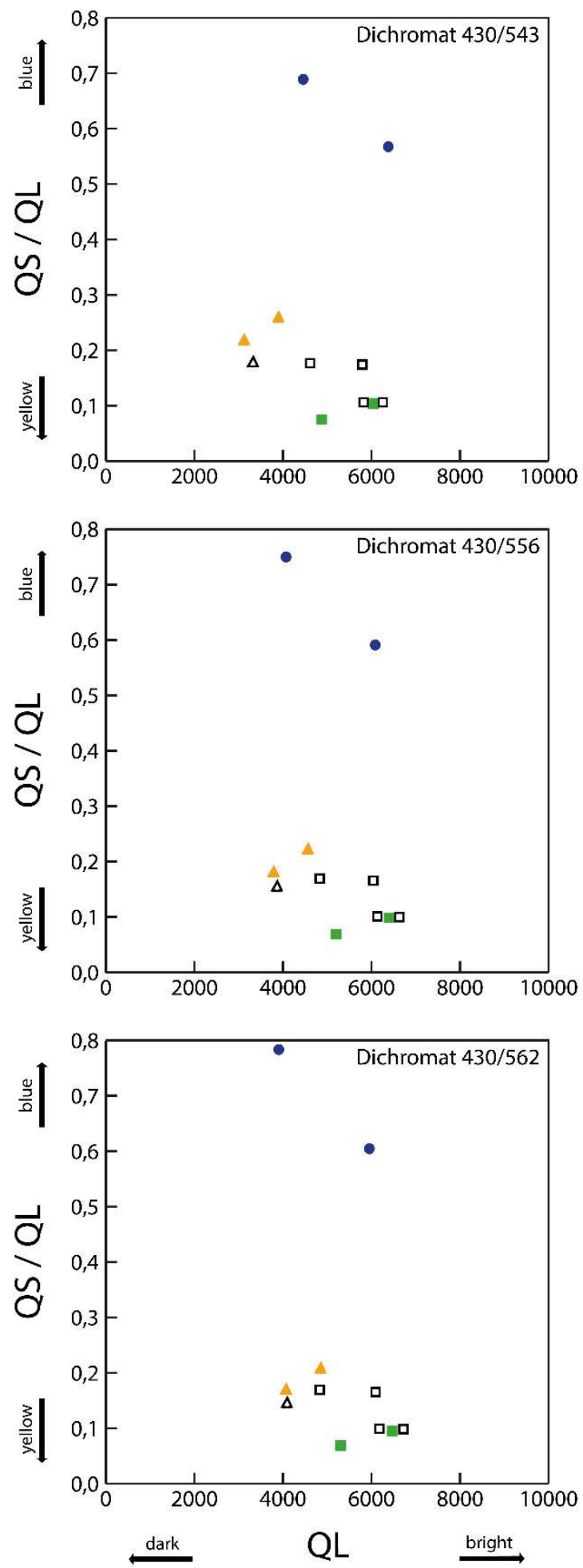

346 Figure 3. Chromaticity/luminance diagrams for three dichromatic phenotypes found in marmosets. Blue

347 (closed circles), orange (closed triangles) and green food targets (closed squares), as well as orange (open

348 triangles) and green (open squares) background elements, are plotted according to how they activate the 349 chromatic blue-yellow (QS/QL) axis and the luminance (QL) axis of marmoset dichromatic vision. 

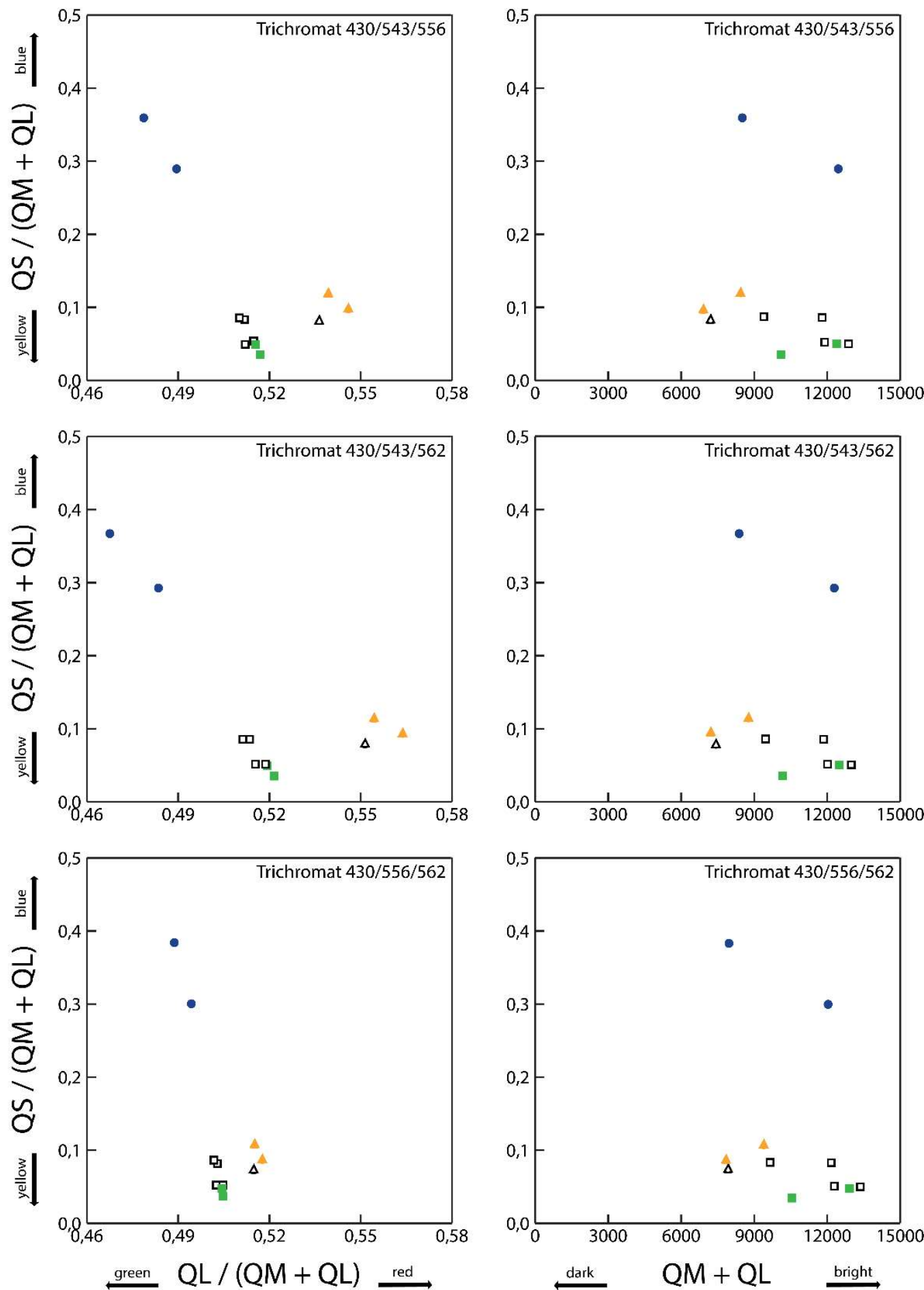

350 Figure 4. Chromaticity/luminance diagrams for three trichromatic phenotypes found in marmosets. Blue

351 (closed circles), orange (closed triangles) and green food targets (closed squares), as well as orange (open

352 triangles) and green (open squares) background elements, are plotted according to how they activate the

353 chromatic red-green $[\mathrm{QL} /(\mathrm{QM}+\mathrm{QL})]$ and blue-yellow $[\mathrm{QS} /(\mathrm{QM}+\mathrm{QL})]$ axes, and the luminance $(\mathrm{QM}+\mathrm{QL})$ axis of marmoset trichromatic vision. 


\subsection{Influence of target shape}

357

Shape of food items significantly influenced foraging time $\left(\mathrm{F}_{1,22}=34.20 ; \mathrm{p}<.001\right.$;

358

$\eta^{2}$ Partial $\left.=0.609\right)$ and percentage of targets captured $\left(F_{1,22}=9.60 ; p=.005 ; \eta^{2}\right.$ Partial $\left.=0.304\right)$,

359 favoring the detection of spherical over cubical targets (Figures 5C and 5D).

360 We also found an interaction between target shape and $\operatorname{sex}\left(\mathrm{F}_{1,22}=5.40 ; \mathrm{p}=.030\right.$;

$361 \eta^{2}$ Partial $=0.197$ ) regarding foraging time, with females investing more time foraging for

362 green cubical food than males (Figure 5D).

363

\subsection{Influence of background chromatic noise}

364 Background chromatic noise had no influence on foraging time invested by females

365 or males $\left(F_{1,22}=0.55 ; p=.463 ; \eta^{2}\right.$ Partial $\left.=0.025\right)$. Yet, concerning the percentage of targets

366 captured, we found an interaction between background chromatic noise and sex

$367\left(\mathrm{~F}_{1,22}=11.50 ; \mathrm{p}=.003 ; \eta^{2}\right.$ Partial $\left.=0.343\right)$. On one hand, females captured orange spherical

368 targets on a dappled green background more frequently than males. On another hand,

369 males significantly captured more orange spherical food items deposited on a dappled

370 green and orange background than on a dappled green background (Figure 5E). 

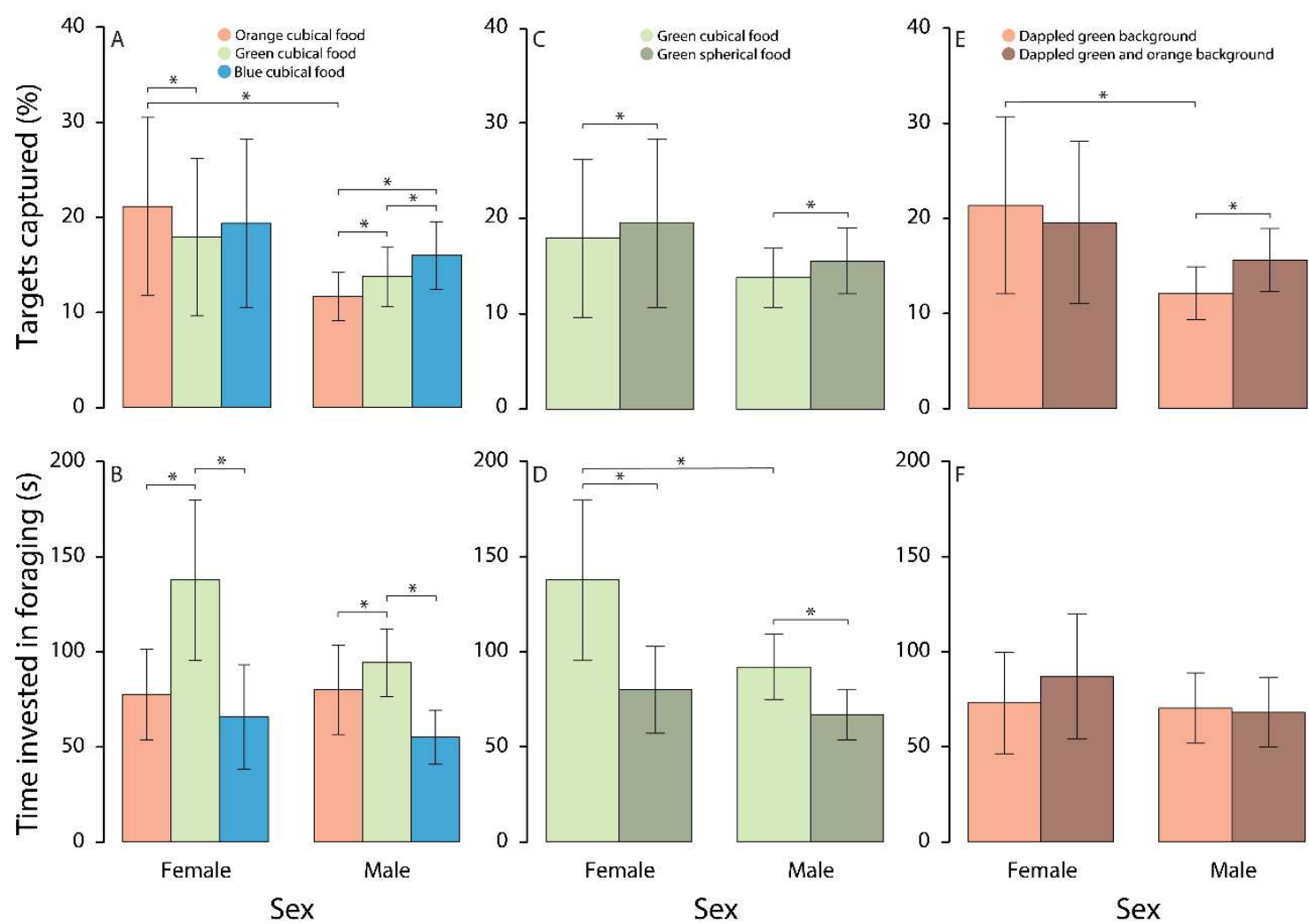

371 Figure 5. Influence of target color, target shape and background chromatic noise on the feeding performance (average foraging time and average percentage of targets captured) of female and male captive marmosets

373 (Callithrix jacchus). Confidence intervals are represented by vertical whiskers. Horizontal bars with asterisks indicate significant differences for $\alpha=.05$.

\section{DISCUSSION}

\subsection{Influence of target color}

Our behavioral results show a clear-cut difference between female and male

subjects' performance and are in line with data modeled by our chromaticity/luminance diagrams, corroborating our first prediction that females should outperform males when searching for orange cubical targets, but not for green or blue cubical food. These results are a clear indication that our female subjects, as a group, indeed behaved as trichromatic individuals, outperforming dichromatic marmoset males in red-green discrimination tasks, as extensively documented by literature for captive marmosets (Caine and Mundy 
385

386

387

2000; Caine et al. 2003; Pessoa et al. 2005b). Although our study subjects were not genotyped with respect to their color vision, we believe differences between female and male performance shown here cannot be chiefly attributed to innate sex differences, instead of color vision phenotype differences, since no difference in female and male performance were found when animals were searching for blue food items (i.e., the positive control condition). In addition, It is important to highlight that, although our chromaticity/luminance diagrams sometimes depict food targets as being darker or lighter than background elements (i.e., overlapping is not perfect), the use of brightness cues by our experimental subjects was almost certainly prevented, once we varied the brightness of our targets and background elements, making luminance cues unreliable.

\subsection{Influence of target shape}

Regarding the influence of shape cues on target detection, we confirmed our second prediction that females and males would benefit from shape cues and segregate green spherical targets much easier than green cubical ones. Unexpectedly, we also found that dichromatic males invested a lower amount of time searching for cryptic targets (i.e., green cubical food), outperforming females. This dichromatic advantage for cryptic food detection contradicts what has been found by psychophysical experiments, that presented human subjects with photos of wild fruits (Melin et al. 2013) and revealed a trichromatic advantage for cryptic fruit detection. Also contradicts a behavioral ecology study conducted in tamarins (Smith et al. 2012), which concluded that trichromats were superior to dichromats at detecting green generalist insects. Furthermore, previous naturalistic studies involving captive marmosets (Caine and Mundy 2000; Caine et al. 2003) have failed to show any significant dichromatic advantage for cryptic food identification. 
410 foraging of colored food items by non-human primates. In theory, regardless of

411 information concerning color, shape cues may be capable of indicating the presence of

412 food (e.g., fruits and insects) in vegetation. When objects are conspicuously colored, the

413 relative importance of shape may decline. However, in the presence of chromatic noise

414 (e.g., disruptive coloration camouflage) or in situations in which coloration conceals

415 targets (e.g., background matching camouflage), shape information should become more

416 significant (Saito et al. 2005). Our results confirm that shape cues are important under

417 seminatural conditions and play a complementary role in food identification. In fact,

418 according to Párraga and collaborators (2002), the shape of fruits closely match those that

419 our visual system finds optimal. In the present study, when we tested the effect of shape

420 cues on food identification, the influence of other visual cues (e.g., color, brightness,

421 texture, gloss, and size) was considered negligible, once they were either controlled (i.e.,

422 stimuli's color, size, texture, and gloss) or randomized (i.e., stimuli's brightness).

\subsection{Influence of background chromatic and achromatic noise}

Presenting a performance consistent with that of trichromats, female marmosets outperformed dichromatic males in tasks involving the detection of orange spherical targets against a dappled green background, confirming our third prediction. However, our data also rejected our fourth prediction that males would outperform females when searching for orange spherical items under camouflaged conditions. Instead, we revealed that camouflage did not influence the performance of female subjects, that captured comparable amounts of orange spherical food against both kinds of backgrounds tested

431 in the present study. Still, in contrast, camouflage exerted a significant impact on male 
432 feeding success. Under the camouflaged condition (i.e., dappled green and orange

433 background) dichromatic males improved their detection of orange spherical targets,

434 when compared to the non-camouflaged condition (i.e., dappled green background),

435 matching females' performance. The influence of a camouflaged background on

436 conspicuous food detection by dichromatic marmosets contradicts what have been

437 presented by a previous study (Caine et al. 2003). Caine and collaborators (2003) found

438 that camouflaged, in comparison to non-camouflaged conditions, significantly reduces

439 feeding performance in trichromatic marmosets, but not dichromatic ones. We believe

440 this discrepancy could be attributable to differences in the spectral features of the

441 background elements used in each study. According to the reflectance spectra of

442 background elements, it appears that Caine's non-camouflaged green background was

443 less variegated (i.e., presented a reduced variance in brightness) when compared to ours,

444 what might have generated lower levels of achromatic noise. Alternatively, our dappled

445 green background must have produced much more achromatic noise, which possibly

446 made tasks more difficult and resemblant to natural challenges.

447 Both of our unexpected results, the dichromatic advantage for cryptic food

448 identification and the dichromatic improvement in conspicuous food detection under

449 camouflaged conditions might be explained by how primate visual systems are organized.

450 In primates, hierarchical processing of chromatic and achromatic information flows in

451 parallel pathways and tend to compete for computational power, in such a way that color

452 processing hampers achromatic processing (including shape information) and vice-versa

453 (Tovée 2008). Consequently, an animal devoid of color vision, or with a reduced color

454 discrimination capability (e.g., dichromat), would reserve most of the processing power

455 allocated to visual processing to its parallel achromatic pathway, improving the detection 
456 of contours, textures, and shapes (Morgan et al. 1992; Saito et al. 2005, 2006).

457 Alternatively, in trichromats, an additional color processing pathway (red-green color 458 opponency), absent in dichromats (Mollon 1989), would demand processing power that 459 would have to be redirected from the achromatic pathway.

460 Background coloration exerts a substantial influence on object identification, given 461 that it can increase target segregation based on their color contrast, as well as promote 462 perceptual confusion, by modifying the color of objects (Shevell and Kingdom 2008) and

463 introducing chromatic and/or achromatic noise (Liebe et al. 2009). Since it supposedly 464 presents less achromatic noise then the dappled green background, the dappled green and 465 orange background must have facilitated the detection of spherical orange targets by 466 dichromatic males based on shape cues, which explains the improvement in male's 467 performance under camouflage conditions. The dichromatic visual system of male marmosets cannot segregate orange and green background elements, so males might have

469 perceived the camouflaged background as roughly homogenous, barely being affected by 470 its chromatic noise, what would allow these dichromatic individuals to effortlessly use 471 the contour of orange spherical targets to segregate them from the cubical background 472 elements. In contrast, the same condition posed a problem to females because, for 473 trichromats, the dappled green and orange background must have significantly enhanced 474 chromatic noise, disturbing shape perception.

475 At first, it might seem unexpected that male dichromats outperformed females when 476 foraging for cryptic food (i.e., green cubical food) under a highly achromatically noisy 477 condition (i.e., dappled green background), since achromatic noise should have adversely 478 affected both male and female performances. Once, due to its four different shades 479 (brightness) of green background elements, the non-camouflaged background must have 
480

481

482

483

484

485

486

487

488

489

490

491

492

493

494

495

496

497

498

499

500

501

appeared extremely heterogeneous to all animals (Párraga et al. 2002). One possible explanation to dichromatic advantage would be that, even though green targets look chromatically cryptic when compared to green background elements, discrete red-green chromatic differences between targets and background elements could have generated enough chromatic noise to affect the discrimination of food by trichromats. Chromatic noise generated by the blue-yellow chromaticity axes of dichromats and trichromats, in turn, would be a shared disadvantage, so it would not be significant for their differential performance.

\section{CONCLUSION}

Here, we confirmed that trichromats are advantageous in orange food detection. Surprisingly, we unveiled a priviously unknown dichromatic advantage for green food identification and found that background color impacts target identification by dichromatic marmosets, but not trichromats. These findings reinforce the idea that visual polymorphism in Neotropical primates is maintained through balancing selection (Hiwatashi et al. 2010), and it would involve multiple advantages and disadvantages of dichromats and trichromats (Pessoa et al. 2014).

Although blue fruits have been reported to comprise the diet of some primate species (Savage et al. 1987; Dominy 2004; Perini et al. 2009), this is only the second Neotropical primate naturalistic study (besides Freitag and Pessoa 2012) to explore the detection of blue food items against a green dappled background. Our data have demonstrated that female and male captive marmosets have no trouble identifying blue food items against a dappled green background. 
In summary, our results show that both color and shape cues are important sources

504 of visual information that influence target discrimination according to the degree of

505 prevailing background noise. However, it is essential to remember that color vision is a

506 complex phenomenon that also depends on other object features and on environmental

507 luminosity, such as stimuli size (Gomes et al. 2005) and ambient light intensity (Pessoa

508 and Freitag 2019). We urge future naturalistic studies to investigate underexplored visual

509 factors such as stimuli's brightness (Hiramatsu et al. 2008), texture, gloss, and size

510 (Valenta et al. 2020).

511

512 6. CRediT AUTHOR STATEMENT

513 Priscilla K.S. Barros: Conceptualization, Methodology, Validation, Investigation,

514 Data curation, Writing - Original Draft, Writing - Review \& Editing, Project

515 Administration. Felipe N. Castro: Formal analysis, Data curation, Writing - Review \&

516 Editing. Daniel M.A. Pessoa: Conceptualization, Methodology, Resources, Writing -

517 Review \& Editing, Visualization, Supervision, Funding acquisition.

\section{ACKNOWLEDGEMENTS}

520 This study was financed in part by the Coordenacao de Aperfeicoamento de Pessoal de

521 Nivel Superior - Brazil (CAPES), Finance Codes 001 and 043/2012, that also provided a

522 M.Sc. Scholarship to P.K.S.B.; by Conselho Nacional de Desenvolvimento Cientifico e

523 Tecnologico - Brazil (CNPQ), Finance Codes 478222/2006-8 and 474392/2013-9, that

524 also provided a Researcher Scholarship to D.M.A.P.; and by Programa de Apoio aos

525 Nucleos de Excelencia - FAPERN/CNPq, Finance Code 25674/2009. The funding 
527 writing or in the decision to submit the article for publication. We would like to thank the

528 Primate Center's staff for animal care and maintenance, specially J.F.V. Coutinho and

529 E.C. Rodrigues. We are also indebted to L.E.F. Carvalho, S.R.N. Silva and S.S. Farias 530 for assisting in data collection.

\section{REFERENCES}

533 Abreu F, Souto A, Bonci DMO, Mantovani V, Pessoa DMA, Schiel N (2019) Detection

534 of insect prey by wild common marmosets: The effect of color vision. Am J Primatol 81:e22963. DOI:10.1002/ajp.22963

536 Altmann J (1974) Observational study of behavior sampling methods. Behaviour 49:227267. DOI:10.1163/156853974X00534

538 Arruda MF, Yamamoto ME, Pessoa DMA, Araujo A (2019) Taxonomy and Natural 539 History. In Marini RP, Wachtman LM, Tardif SD, Mansfield K, Fox JG (eds.) The

540 Common Marmoset in Captivity and Biomedical Research (American College of 541 Laboratory Animal Medicine). Academic Press, New York. pp 3-15. DOI:10.1016/B978-0-12-811829-0.00001-7

543 Bompas A, Kendall G, Sumner P (2013) Spotting fruit versus picking fruit as the selective 544 advantage of human colour vision. i-Perception 4:84-94. DOI:10.1068/i0564

545 Bowmaker JK (1998) Evolution of colour vision in vertebrates. Eye 12:541-547. $546 \quad$ DOI: $10.1038 /$ eye.1998.143

547 Caine NG, Mundy NI (2000) Demonstration of a foraging advantage for trichromatic 548 marmosets (Callithrix geoffroyi) dependent on food color. Proc Biol Sci 267:439-444. DOI:10.1098/rspb.2000.1019 
550 Caine NG, Surridge AK, Mundy NJ (2003) Dichromatic and trichromatic Callithrix 551 geoffroy differ in relative foraging ability for red-green color camouflaged and non552 camouflaged food. Int J Primatol 24:1163-1175. $553 \quad$ DOI:10.1023/B:IJOP.0000005985.18112.25

554 Carvalho LS, Pessoa DMA, Mountford JK, Davies WI, Hunt DM (2017) The genetic and 555 evolutionary drives behind primate color vision. Front Ecol Evol 5:34. DOI:10.3389/fevo.2017.00034

557 Cowey A, Heywood CA (1995) There's more to colour than meets the eye. Behav Brain Res 71:89-100. DOI:10.1016/0166-4328(95)00046-1

559 De Moraes PZ, Diniz P, Spyrides MHC, Pessoa DMA (2021) The effect of pelage, 560 background, and distance on predator detection and the evolution of primate color vision. Am J Primatol 83:e23230. DOI:10.1002/ajp.23230

562 Dominy NJ (2004) Color as an indicator of food quality to anthropoid primates:

563 Ecological evidence and an evolutionary scenario. In Ross C, Kay RF (eds.) 564 Anthropoid Origins: New Visions. Kluwer Academic, New York. pp 615-644. 565 DOI:10.1007/978-1-4419-8873-7_22

566 Dominy NJ, Lucas PW (2001) Ecological importance of trichromatic vision to primates. Nature 410:363-365. DOI:10.1038/35066567

568 Dominy NJ, Garber PA, Bicca-Marques JC, Azevedo-Lopes MAO (2003) Do female 569 tamarins use visual cues to detect fruit rewards more successfully than do males? Anim $570 \quad$ Behav 66:829-837. DOI: 10.1006/anbe.2003.2288

571 Freitag FB, Pessoa DMA (2012) Effect of luminosity on color discrimination of 572 dichromatic marmosets (Callithrix jacchus). JOSA 29:A216-A222. 
574 Gomes UR, Pessoa DMA, Suganuma E, Tomaz C, Pessoa VF (2005) Influence of stimuli 575 size on color discrimination in Capuchin monkeys. Am J Primatol 67:437-446. DOI: 10.1002/ajp.20198

577 Heesy CP, Ross CF (2001) Evolution of activity patterns and chromatic vision in 578 primates: morphometrics, genetics and cladistics. J Human Evol 40:111-149. $579 \quad$ DOI:10.1006/jhev.2000.0447]

580 Hiramatsu C, Melin AD, Aureli F, Schaffner CM, Vorobyev M, Matsumoto Y, 581 Kawamura S (2008) Importance of Achromatic Contrast in Short-Range Fruit 582 Foraging of Primates. PLOS ONE 3:e3356. DOI10.1371/journal.pone.0003356

583 Hiwatashi T, Okabe Y, Tsutsui T, Hiramatsu C, Melin AD, Oota H, Schaffner CM, Aureli 584 F, Fedigan LM, Innan H, Kawamura S (2010) An explicit signature of balancing 585 selection for color-vision variation in New World monkeys. Mol Biol Evol 27:453464. DOI:10.1093/molbev/msp262

587 Hunt DM, Williams AJ, Bowmaker JK, Mollon JD (1993) Structure and evolution of the 588 polymorphic photopigment gene of the marmoset. Vision Res 33:147-154. DOI:10.1016/0042-6989(93)90153-n

590 Jacobs GH (2007) New World monkeys and color. Int J Primatol 28:729-759. DOI:10.1007/s10764-007-9168-y

592 Jacobs GH (2010) Recent progress in understanding mammalian color vision. Ophthal Physl Opt 30:422-434. DOI:10.1111/j.1475-1313.2010.00719.x

594 Jacobs GH (2018) Photopigments and the dimensionality of animal color vision. Neurosci 595 Biobehav R 86:108-130. DOI:10.1016/j.neubiorev.2017.12.006

596 Kay RF (1984) On the use of anatomical features to infer foraging behavior in extinct 597 primates. In Rodman PS, Cant JGH, (eds.) Adaptations for foraging in nonhuman 
598 primates. Columbia University Press, New York. pp 21-53. DOI:10.7312/rodm90184$599 \quad 003$

600 Kay RF, Williams BA, Anaya F (2002) The adaptations of Branisella boliviana, the 601 earliest South American monkey. In Plavcan JM, Kay RF, Jungers WL, van Schaik 602 CP (eds.). Reconstructing behavior in the primate fossil record. Kluwer-Plenum, New 603 York. pp 339-370. DOI:10.1007/978-1-4615-1343-8_9

604 Kawamura S, Hirai M, Takenaka O, Radlwimmer FB, Yokoyama S (2001) Genomic and 605 spectral analyses of long to middle wavelength-sensitive visual pigments of common 606 marmoset(Callithrix jacchus). Gene 269:45-51. DOI:10.1016/S0378-1119(01)00454$607 \quad 1$

608 Liebe S, Fischer E, Logothetis NK, Rainer G (2009) Color and shape interactions in the 609 recognition of natural scenes by human and monkey observers. J Vis 9:1-16. $610 \quad$ DOI: $10.1167 / 9.5 .14$

611 Leonhardt SD, Tung J, Camden JB, Leal M, Drea CM (2009) Seeing red: behavioral 612 evidence of trichromatic color vision in strepsirrhine primates. Behav Ecol 20:1-12. 613 DOI:10.1093/beheco/arn106

614 Maia R, Gruson H, Endler JA, White TE (2019) pavo 2: New tools for the spectral and 615 spatial analysis of colour in R. Methods Ecol Evol 10:1097-1107. DOI:10.1111/2041$616 \quad 210 X .13174$

617 Mantovani V, Hauzman E, Corredor VH, Goulart PRK, Galvão O, Talebi M, Pessoa 618 DMA, Soares JGM, Fiorani M, Gattass R, Ventura DF, Bonci DMO (2020) Genetic 619 variability of the sws1 cone opsin gene among New World monkeys. Am J Primatol 620 82:e23199. DOI:10.1002/ajp.23199 
621 Melin AD, Fedigan ML, Hiramatsu C, Sendall CL, Kawamura S (2007) Effects of colour 622 vision phenotype on insect capture by a free-ranging population of white-faced 623 capuchins, Cebus capucinus. Anim Behav 205-214. 624 DOI:10.1016/j.anbehav.2006.07.003

625 Melin AD, Kline DW, Hickey C, Fedigan ML (2013) Food search through the eyes of a 626 monkey: A functional substitution approach for assessing the ecology of primate color 627 vision. Vision Res 86:87-96. DOI:10.1016/j.visres.2013.04.013

628 Melin AD, Chiou KL, Walco ER, Bergstrom ML, Kawamura S, Fedigan LM (2017) 629 Trichromacy increases fruit intake rates of wild capuchins (Cebus capucinus imitator). $630 \quad$ PNAS 114:10402-10407. DOI:10.1073/pnas.1705957114

631 Mollon JD (1989) “Tho'she kneel'd in that place where they grew...”The uses and origins 632 of primate colour vision. J Exp Biol 146:21-38.

633 Moreira LAA, De Oliveira DGR, De Sousa MBC, Pessoa DMA (2015) Parturition 634 Signaling by Visual Cues in Female Marmosets (Callithrix jacchus). PLOS ONE 635 10:e0129319. DOI:10.1371/journal.pone.0129319

636 Morgan MJ, Adam A, Mollon JD (1992) Dichromats detect colour-camouflaged objects 637 that are not detected by trichromats. Proc R Soc Lond B 248:291-295. $638 \quad$ DOI: $10.1098 /$ rspb.1992.0074

639 Párraga CA, Troscianko T, Tolhurst DL (2002) Spatiochromatic properties of natural 640 images and human vision. Curr Biol 12:483-487. DOI:10.1016/S0960$641 \quad 9822(02) 00718-2$

642 Perini ES, Pessoa VF, Pessoa DMA (2009) Detection of fruit by the Cerrado's marmoset 643 (Callithrix penicillata): modeling color signal for different background scenarios and 644 ambient light intensities. J Exp Zool 311A:289-302. DOI:10.1002/jez.531 
645 Pessoa DMA, Cunha JF, Tomaz C, Pessoa VF (2005a) Colour discrimination in the black646 tufted-ear marmoset (Callithrix penicillata): ecological implications. Folia Primatol 647 76:125-134. DOI:10.1159/000084375

648 Pessoa DMA, Freitag FB (2019) Effect of luminosity on color discrimination of 649 dichromatic marmosets (Callithrix jacchus): erratum. JOSA 36:508-509. 650 DOI:10.1364/JOSAA.36.000508

Pessoa DMA, Tomaz C, Pessoa VF (2005b) Color vision in marmosets and tamarins: behavioral evidence. Am J Primatol 67:487-495. DOI:10.1002/ajp.20202 The adaptive value of primate color vision for predator detection. Am J Primatol published online. DOI:10.1002/ajp.22264

R Core Team (2020). R: A language and environment for statistical computing. R Foundation for Statistical Computing, Vienna. URL https:/www.R-project.org/ Fruits, foliage and the evolution of primate color vision. Philos Trans R Soc Lond B Biol Sci 356:229-283. DOI:10.1098/rstb.2000.0773

661 Saito A, Mikami A, Kawamura S, Ueno Y, Hiramatsu C, Widayati KA, Surybroto B, 662 Teramoto M, Mori Y, Nagano K, Fujita K, Kuroshima H, Hasegawa T (2005) 663 Advantage of dichromats over trichromats in discrimination of color-camuflaged 664 stimuli in non-human primates. Am J Primatol 67:425-436. DOI:10.1002/ajp.20197

665 Saito A, Mikami A, Hosokawa T, Hasegawa T (2006) Advantage of dichromats over 666 trichromats in discrimination of color-camouflaged stimuli in humans. Percept Motor 667 Skill 102:3-12. DOI:10.2466/pms.102.1.3-12 
668 Savage A, Dronzek LA, Snowdon CT (1987) Color discrimination by the cotton-top 669 tamarin (Saguinus oedipus oedipus) and its relation to fruit coloration. Folia Primatol 49:57-69. DOI:10.1159/000156309

671 Shevell SK, Kingdom FA (2008) Color in complex scenes. Annu Rev Psychol 59:143166. DOI:10.1146/annurev.psych.59.103006.093619

673 Shyue SK, Boissinot S, Schneider H, Sampaio I, Schneider MP, Abee CR, Williams L, 674 Hewett-Emmett D, Sperling HG, Cowing JA, Dulai KS, Hunt DM, Li WH (1998) 675 Molecular genetics of spectral tuning in New World monkey color vision. J Mol Evol 46:697-702. DOI:10.1007/PL00006350

677 Smith AC, Buchanan-Smith HM, Surridge AK, Osorio D, Mundy NI (2003) The effect 678 of color vision status on the detection and selection of fruits by tamarins (Saguinus 679 spp.). J Exp Biol 206:3159-3165. DOI:10.1242/jeb.00536

680 Smith AC, Surridge AK, Prescot MJ, Osorio D, Mundy NI, Buchanan-Smith HM (2012) 681 Effect of colour vision status on insect prey capture efficiency of captive and wild 682 tamarins (Saguinus spp.). Anim 83:479-486. 683 DOI:10.1016/j.anbehav.2011.11.023

684 Sumner P, Mollon JD (2000) Catarrhine photopigments are optimized for detecting 685 targets against a foliage background. J Exp Biol 203:1963-1986

686 Surridge AK, Mundy NJ (2002) Trans-specific evolution of opsin alleles and the 687 maintenance of trichromatic colour vision in Callitrichine primates. Mol Ecol 11:2157688 2169. DOI:10.1046/j.1365-294X.2002.01597.x

689 Tovée MJ (2008) An introduction to the visual system. Cambridge University Press, 690 Cambridge. 212 p. 
691 Tovée MJ, Bowmaker JK, Mollon JD (1992) The relationship between cone pigments 692 and behavioural sensitivity in a New World monkey (Callithrix jacchus jacchus). 693 Vision Res 32:867-878. DOI:10.1016/0042-6989(92)90029-I

694 Travis DS, Bowmaker, JK, Mollon, JD (1988) Polymorphism of visual pigments in a 695 Callitrichid monkey. Vision Res 28:481-490. DOI:10.1016/0042-6989(88)90170-8 696 Valenta K, Daegling DJ, Nevo O, Ledogar J, Sarkar D, Kalbitzer U, Bortolamiol S, Omeja 697 P, Chapman CA, Ayasse M, Kay R, Williams B (2020) Fruit Selectivity in Anthropoid 698 Primates: Size Matters. Int J Primatol 41:525-537. DOI:10.1007/s10764-020-00158-3

699 Wichmann FA, Sharpe LT, Gegenfurtner KR (2002) The contributions of color to 700 recognition memory for natural scenes. J Exp Psychol Learn Mem Cogn 28:509-520. $701 \quad$ DOI: $10.1037 / 0278-7393.28 .3 .509$

702 Wilder, H., Grünert, U., Lee, B., \& Martin, P. (1996). Topography of ganglion cells and 703 photoreceptors in the retina of a New World monkey: The marmoset Callithrix 704 jacchus. Visual Neurosci, 13(2), 335-352. DOI10.1017/S0952523800007586

705 Yamashita N, Stoner KE, Riba-Hernández P, Dominy NJ, Lucas PW (2005) Light levels 706 used during feeding by primate species with different color vision phenotypes. Behav Ecol Sociobiol 58:618-629. DOI:10.1007/s00265-005-0936-4 